\title{
INFLUÊNCIA DO DIMENSIONAMENTO DA EQUIPE DE ENFERMAGEM NA QUALIDADE DO CUIDADO AO PACIENTE CRÍTICO
}

\author{
Gelena Lucinéia Gomes da Silva Versaํ, Kelly Cristina Inoue², Anair Lazzari Nicola², Laura Misue Matsuda
}

\footnotetext{
${ }^{1}$ Mestranda em Enfermagem pela Universidade Estadual de Maringá (UEM). Enfermeira Coordenadora das Comissões Intrahospitalares do Hospital Universitário do Oeste do Paraná. Paraná, Brasil. E-mail: gelenaenfermagem@yahoo.com.br

${ }^{2}$ Mestre em Enfermagem. Enfermeira da Unidade de Terapia Intensiva Adulto do Hospital Universitário de Maringá. Docente do Curso de Enfermagem da Faculdade Uningá. Paraná, Brasil. E-mail: kellyelais@hotmail.com

${ }^{3}$ Doutora em Enfermagem. Docente da Universidade Estadual do Oeste do Paraná. Paraná, Brasil. E-mail: anairln@yahoo.com.br

${ }^{4}$ Doutora em Enfermagem. Docente da UEM. Paraná, Brasil. E-mail: 1mmatsuda@uem.br
}

RESUMO: Estudo na modalidade Revisão Integrativa, com objetivo de analisar a influência do dimensionamento do pessoal de enfermagem intensivista na qualidade do cuidado ao paciente crítico adulto. Foram incluídas apenas publicações científicas, das principais bases eletrônicas, veiculadas em periódicos de acesso livre e eletrônico, referentes à última década (janeiro de 2000 a janeiro de 2010), nos idiomas inglês, português e espanhol. Obteve-se um total de 10 publicações, dentre as quais três avaliaram a incidência de mortalidade e extubação acidental no pós-operatório; três avaliaram os reflexos no cuidado resultantes da redução no número de enfermeiros; dois avaliaram o desenvolvimento de infecções; e um, respectivamente, avaliou a incidência de quedas e de pneumonia associada à ventilação mecânica. Os estudos indicam relação entre o subdimensionamento de trabalhadores da enfermagem e o aumento nas taxas de infecções, mortalidade, quedas, pneumonia associada à ventilação mecânica e extubação acidental.

DESCRITORES: Downsizing organizacional. Enfermagem. Qualidade da assistência à saúde. Unidades de terapia intensiva.

\section{INFLUENCE OF DIMENSIONING THE NURSING STAFF ON THE QUALITY OF CARE OF THE CRITICAL PATIENT}

\begin{abstract}
The objective of this Integrative Review is to analyze the influence of dimensioning of intensive care nursing staff on the quality of critical adult patient care delivered. Only scientific publications from major electronic databases published in open access journals online during the last decade (January 2000 to January 2010) in English, Portuguese, and Spanish were included. A total of 10 publications were thus obtained, among which three evaluated the incidence of mortality and post-operational accidental extubation, three evaluated the reflections on care resulting from reduced nursing staff; two rated the development of infections; and one estimated the incidence of falls and ventilator-associated pneumonia. These studies indicate the relationship between sub-dimensioning nursing workers and increased infection rates, mortality, falls, ventilator-associated pneumonia, and accidental extubation.
\end{abstract}

DESCRIPTORS: Personnel downsizing. Nursing. Quality of health care. Intensive care units.

\section{INFLUENCIA DEL CÁLCULO DEL PERSONAL DEL EQUIPO DE ENFERMERÍA EN LA CALIDAD DE LA ATENCIÓN AL PACIENTE EN ESTADO CRÍTICO}

RESUMEN: Se trata de un estudio de revisión integral, con el fin de analizar la influencia del cálculo del personal de enfermería intensivista en lo que se refiere a la calidad de la atención al paciente adulto en estado crítico. Sólo se incluyeron las publicaciones científicas, de las principales bases de datos electrónicas, difundidas en acceso abierto y electrónico, referentes a la última década (enero de 2000 hasta enero de 2010), en inglés, portugués y español. Se obtuvo un total de diez publicaciones, tres de ellas, evaluaron la incidencia de mortalidad y la extubación accidental en el postoperatorio, tres calificaron los reflejos en la atención derivada de la reducción en el número de enfermeras, dos calificaron la aparición de infecciones, y en un artículo se evaluó la incidencia de caídas y de neumonía asociada al ventilador. Los estudios indican la relación entre la infravaloración del personal de enfermería y un aumento en las tasas de infección, mortalidad, caídas, neumonía asociada al ventilador y la extubación accidental.

DESCRIPTORES: Reducción de personal. Enfermería. Calidad de la atención de salud. Unidades de terapia intensiva. 


\section{INTRODUÇÃO}

Nas últimas décadas, a globalização tem impulsionado mudanças econômicas, políticas, sociais e culturais nos países e nas organizações, devido, em parte, à incorporação crescente de novas tecnologias e da integração comercial em nível mundial. Embora em ritmo menos acelerado, o setor de saúde também tem acompanhado esse processo, na tentativa de se adequar às novas exigências da população e obter mais qualidade e produtividade.

Nos hospitais, a preocupação com os custos e investimentos à assistência à saúde se deve, principalmente, às unidades críticas, pois demandam maior ônus institucional, em razão dos aspectos relacionados à estrutura - recursos físicos, materiais, tecnológicos e/ou humanos - indispensáveis à prestação de seus serviços.

Reconhece-se que em uma instituição hospitalar a Unidade de Terapia Intensiva (UTI), por atender a populações com necessidades terapêuticas especiais, as quais demandam alta tecnologia e recursos humanos em larga escala, é o local no qual ocorrem os maiores gastos para o atendimento. Desse modo, é fundamental que, associada à redução de custos e ao aumento da produtividade, a busca pela qualidade vise à promoção/manutenção da segurança do cliente. ${ }^{1}$

A qualidade é entendida como o uso eficiente dos recursos físicos e humanos, com o mínimo de risco ao cliente e alto grau de satisfação aos usuários. ${ }^{2}$ Nesse contexto, para o alcance da qualidade do cuidado, a otimização dos recursos existentes em UTI e a alocação de pessoal de enfermagem qualificado para a assistência são quesitos fundamentais, ${ }^{3}$ uma vez que a equipe de enfermagem superestimada implica em alto custo e, quando reduzida, pode ocasionar menor eficiência do serviço prestado. ${ }^{4}$

Ressalta-se que o número de trabalhadores de enfermagem e sua qualificação por categoria profissional devem ser previstos pelo enfermeiro, ${ }^{5}$ por meio da metodologia do dimensionamento de pessoal de enfermagem. ${ }^{6}$ Tal abordagem consiste em um instrumento gerencial para o planejamento da assistência de qualidade, em razão de auxiliar na adequação do quadro de pessoal às necessidades da clientela e às características do Serviço de Enfermagem e da instituição.
Ao ponderar as consequências do subdimensionamento da equipe de enfermagem que atua em UTI-Adulto (UTI-A) e mediante a importância do número adequado de trabalhadores de enfermagem para a qualidade do cuidado, questiona-se: Considerando as publicações nacionais e internacionais, como se apresenta a relação dimensionamento da equipe de enfermagem e qualidade do cuidado? Quais ocorrências são atribuídas ao dimensionamento inadequado da equipe de enfermagem intensivista?

Para responder às questões enunciadas, foi proposta a realização do estudo, que teve como objetivo: Analisar a influência do dimensionamento do pessoal de enfermagem intensivista na qualidade do cuidado ao paciente crítico adulto.

\section{METODOLOGIA}

Estudo do tipo Revisão Integrativa, composto de seis etapas, ${ }^{7}$ a seguir descritas:

Etapa 1: Tema central: Influência do dimensionamento da equipe de enfermagem na qualidade do cuidado em UTI-A e Questão norteadora: Quais são as consequências à qualidade do cuidado ao paciente crítico devido o dimensionamento inadequado da equipe de enfermagem intensivista adulto?

Etapa 2: Critérios de inclusão - artigos científicos, veiculados em periódicos de acesso livre e eletrônico, publicados na última década (janeiro de 2000 a janeiro de 2010), nos idiomas inglês, português e espanhol, obtidos nas bases de dados eletrônicas: LILACS; Biblioteca Virtual em Saúde (BVS/Bireme); Scopus; Global Health Library e ScienceDirect; localizados ao utilizar os termos de saúde Downsizing Organizacional*, qualidade da assistência à saúde, enfermagem e unidades de terapia intensiva; padronizados pelos Descritores em Ciência da Saúde (DeCS/Bireme), em língua vernácula, bem como seus congêneres (inglês e espanhol).

Etapa 3: Extração das informações e/ou dados estruturais (autor; título; ano; objetivo; metodologia e resultados), os quais, posteriormente, foram compilados em planilhas do programa Microsoft Office Excel ${ }^{\circledR}$.

Etapa 4: Análise dos resumos dos artigos localizados, com exclusão das publicações que não aten-

\footnotetext{
* Downsizing Organizacional é definido pelo DeCS/Bireme como sinônimo de: Downsizing, Ajuste, Redimensionamento, Dimensionamento, Enxugamento e Redução de pessoal e, Redução gerencial no uso de recursos humanos.
} 
deram ao tema central e/ou objetivos da pesquisa, conforme consta na Figura 1.

Etapa 5: Avaliação na íntegra, dos artigos selecionados na etapa 4 . A pós a análise profícua, incluíram-se apenas as publicações que realizaram avaliação/ análise/discussão da influencia do dimensionamento da equipe de enfermagem intensivista na qualidade do cuidado ao paciente crítico adulto.

Etapa 6: Agrupamento dos resultados de acordo com os efeitos/complicações do dimensiona- mento na qualidade dos cuidados prestados em UTI-A. Posteriormente, realizou-se análise estatística simples desses dados (frequência absoluta e percentual).

\section{RESULTADOS E DISCUSSÃO}

Foram encontradas 177 publicações, dessas 167 foram excluídas por não atenderem a todos os critérios de inclusão.

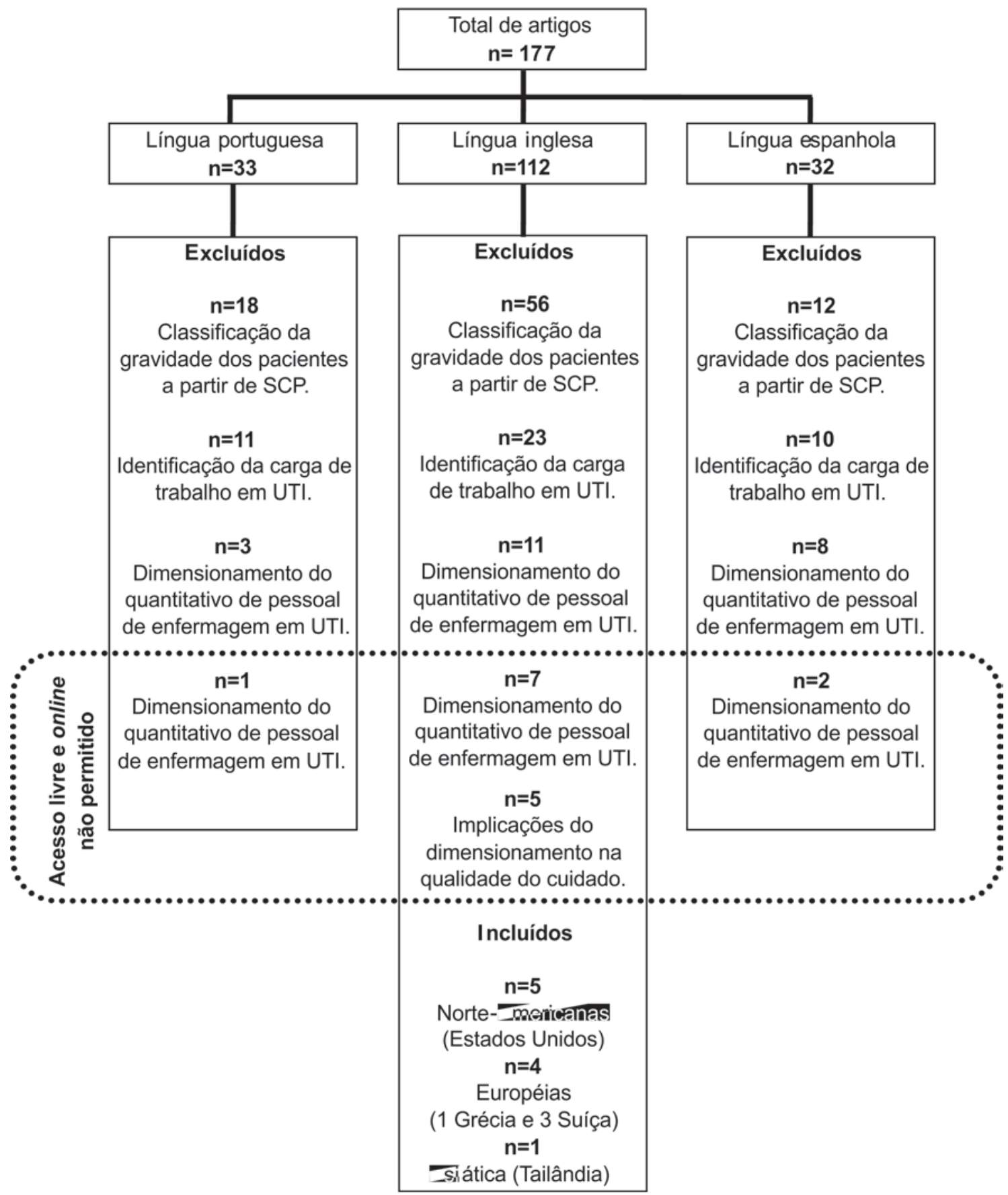

Figura 1 - Esquema de seleção das publicações, de acordo com o tema central e continente/país de origem 
Conforme consta na figura 1 , as pesquisas de maior frequência contemplavam o Sistema de Classificação de Paciente (SCP) $(48,6 \%)$; identificação da carga de trabalho $(24,8 \%)$ e o dimensionamento de pessoal de enfermagem $(18,1 \%)$. A influência do dimensionamento da equipe de enfermagem sobre a qualidade do cuidado totalizou apenas 8,5\% e, destes 2,8\% foram excluídos por não se encontrarem disponíveis por acesso livre online (figura 1).

A predominância das produções acerca de SCP e a identificação de carga de trabalho relacionam-se à insuficiência quantitativa de recursos humanos na enfermagem em nível nacional e internacional, devido à crise econômica nos serviços de saúde, principalmente nos anos de 1990. ${ }^{8}$ Já os trabalhos sobre a qualidade do cuidado na área de enfermagem passaram a ser priorizados somente a partir século XXI, especialmente em UTI, em razão dos gastos expressivos desta unidade, a qual demanda implantação de sistemas de controle do quantitativo de pessoal simultâneo à manutenção da qualidade da assistência. ${ }^{9}$

Vale ressaltar na figura 1, o número de publicações na língua inglesa $(68,9 \%)$ e o fato de que todos os artigos incluídos neste estudo ocorreram nesse idioma. Desse total, $50 \%$ eram de origem norte-americana.

Destaca-se ainda, que as produções latinas, em geral, são em menor proporção e contribuem com apenas 1,4\% das publicações científicas globais. Os Estados Unidos da América (EUA) publicam $35,8 \%$ e a União Europeia 27,7\%. ${ }^{10}$

No tocante às 10 publicações incluídas neste estudo, todas utilizaram como norte metodológico o método quase-experimental. Como estratégia de análise, os estudos utilizaram a avaliação e a comparação entre dois grupos semelhantes assistidos em UTI, sete pesquisas ${ }^{11-17}$ avaliaram os resultados ao nível da qualidade hospitalar e/ou da qualidade do cuidado de enfermagem entre um grupo atendido por uma equipe de enfermagem subestimada e outro que foi atendido por uma equipe cuja adequação quantitativa condizia com os padrões de proporção de enfermagem por paciente preconizados pela British Association of Critical Care Nurses ${ }^{18}$ ou pela European Society of Intensive Care Medicine. ${ }^{19}$

Outra estratégia utilizada por três estudos ${ }^{20-22}$ foi quanto à avaliação dos resultados ao nível da qualidade hospitalar e/ou da qualidade do cuidado de enfermagem quando se alterava apenas o quantitativo do profissional enfermeiro, deno- minado pelos norte-americanos como enfermeiros registrados (Registered nurse-RN). Também nesses estudos, os autores utilizaram como padrão as recomendações dos órgãos antes mencionados.

Quanto aos efeitos do dimensionamento na qualidade do cuidado: três estudos avaliaram a incidência de mortalidade e de extubação acidental em pacientes pós-operatórios; ${ }^{11-13}$ três avaliaram os reflexos no cuidado ao se reduzir o número de enfermeiros (RN); ${ }^{20-22}$ dois avaliaram o desenvolvimento de infecções hospitalares; ${ }^{15-16} \mathrm{um}$ avaliou a incidência de quedas e ${ }^{14} \mathrm{um}$ analisou a incidência de Pneumonia Associada à Ventilação mecânica (PAV). ${ }^{17}$

Os estudos com pacientes cirúrgicos, ${ }^{11-13}$ desenvolvidos em UTIs dos EUA, foram realizados no período pós-operatório de ressecção esofágica, hepactetomia e cirurgia abdominal.

Constatou-se nos pacientes dos estudos americanos, em pós-operatório, déficit na qualidade da assistência quando atendidos por uma equipe subestimada, destacando-se: alta taxa de extubação (10,8\%); aumento da taxa em $21 \%$ de necessidade de reintubação por extubação acidental e; aumento de $43 \%$ das complicações médicas (infecção hospitalar). Assim, os resultados indicam que o quantitativo de pessoal de enfermagem pode influenciar as taxas de mortalidade e riscos de complicações aos pacientes cirúrgicos graves, principalmente, devido à extubação acidental. ${ }^{11-13}$

A retirada não planejada de tubo endotraqueal e a necessidade de reintubação acarretam em sérias consequências para o paciente, dentre as quais constam o aumento do período de Ventilação Mecânica (VM) e de internação, maior exposição à hipoxemia, atelectasia, PAV e instabilidade hemodinâmica, o que, consequentemente, aumenta os riscos de sequelas e óbitos. ${ }^{23}$

Além da extubação acidental, outro incidente grave em UTI se refere à queda, avaliada em um estudo norte-americano, ${ }^{17}$ desenvolvido com 45 pacientes, no qual se detectou um aumento de $7 \%$ de quedas para pacientes atendidos por uma equipe subestimada. Neste estudo estadunidense $^{14}$ a queda culminou em $32 \%$ de complicações relacionadas ao aumento do tempo de internação, do custo do tratamento, de dor e de desconforto ao paciente.

A ocorrência de eventos iatrogênicos ou incidentes negativos, como é o caso das quedas, são episódios potencialmente prejudiciais ao paciente crítico, porque podem causar danos/injúrias de 
qualquer gravidade - inclusive irreversíveis e fatais - acometendo a segurança e a manutenção da qualidade assistencial.

Dentre os eventos iatrogênicos, a infecção hospitalar também se destaca como um evento grave, haja vista que é uma das principais causas de óbito em UTI. ${ }^{15}$

Com o escopo de investigar os fatores desencadeantes das infecções em unidades críticas, dois estudos europeus ${ }^{15,16}$ foram desenvolvidos para avaliar a influência do dimensionamento nas taxas de infecções hospitalares. A primeira pesquisa, desenvolvida em Genebra, ${ }^{15}$ constatou aumento de $50 \%$ de risco de infecção para pacientes atendidos por número de pessoal de enfermagem subestimado e a segunda, realizada na Europa, ${ }^{16}$ detectou $30 \%$ de redução de infecções hospitalares entre aqueles que foram atendidos por um quantitativo de enfermagem adequado.

A infecção hospitalar é um agravante à segurança do paciente grave, pois ocasiona maior tempo de internação, maiores taxas de morbidade e de mortalidade,,$^{24}$ sendo assim, o controle das infecções hospitalares, além de atender às exigências econômicas, legais e éticas, é também requisito para um cuidado seguro.

No enfoque da garantia e manutenção da qualidade da assistência ao paciente crítico e frente à gravidade do quadro infeccioso por ventilação mecânica, a PAV tem se tornado foco de preocupação por parte da equipe multidisciplinar de UTI e de órgãos assessores, para o controle de infecções hospitalares. Isso porque, essa é uma das principais causas de complicações nesse serviço. ${ }^{17}$

$\mathrm{O}$ impacto da PAV sobre as condições clínicas e no prognóstico de pacientes em UTI motivou os pesquisadores suíços ${ }^{17}$ a avaliarem exclusivamente a influência do quantitativo do pessoal de enfermagem sobre o desenvolvimento de PAV nas UTIs de um hospital em Genebra. Os resultados demonstraram que se o cuidado prestado pela equipe de enfermagem fosse realizado por número adequado de profissionais, o risco de PAV diminuiria em 53\%.

A adequação quantitativa dos profissionais de enfermagem resulta em promoção e/ou manutenção da segurança e melhor qualidade do cuidado, principalmente, em UTI, porque se o risco de PAV decresce quando o indivíduo é cuidado por número suficiente de profissionais de enfermagem, haverá menor risco de agravos decorrentes desse processo infeccioso.
Ao considerar a qualificação dos membros que compõem a equipe de enfermagem de UTI, foram realizadas três pesquisas: uma asiática, na Tailândia, ${ }^{20}$ outra nos EUA ${ }^{21}$ e a terceira, na Europa, mais especificamente na Grécia, ${ }^{22}$ com o objetivo de avaliar os resultados do cuidado, ao ser alterada a proporção de enfermeiros (RN) na equipe de enfermagem.

O estudo tailandês apresentou dados de redução dos índices de extubação, de quedas e de úlceras de pressão, quando à equipe de enfermagem foi acrescido maior número de enfermeiros. ${ }^{20}$ A pesquisa norte-americana, ${ }^{21}$ ao dimensionar a equipe de enfermagem com $70 \%$ de $\mathrm{RN}$, obteve redução nas taxas de infecções, de PAV e de custos hospitalares. Já o estudo europeu, ${ }^{22}$ ao reduzir a proporção de RN na equipe de UTI, obteve aumento das taxas de mortalidade.

As pesquisas estrangeiras referidas - asiática, americana e europeia - focam a importância do profissional enfermeiro no cuidado ao paciente crítico e isso é um tema de grande relevância porque o atendimento prestado em quantidade e qualidade adequadas a essa clientela possibilita cuidados especializados e sistematizados. ${ }^{25}$

No contexto da saúde, a equipe multiprofissional, com destaque à equipe de enfermagem, é fundamental à manutenção do cuidado seguro e de qualidade. Nesse sentido, o enfermeiro, o profissional que coordena essa equipe e gerencia o processo de cuidado ao paciente crítico ${ }^{25}$ deve ser suprido em termos de número e qualificação para exercer a sua função.

A importância dos profissionais de enfermagem foi ressaltada nos 10 estudos analisados que, por meio de dados relacionados ao dimensionamento inadequado de profissionais de enfermagem em UTI e sua influência negativa na qualidade da assistência, apontam à necessidade do dimensionamento de enfermagem nesse serviço.

\section{CONCLUSÃO}

Os estudos analisados indicam a relação entre o quantitativo de enfermagem subestimado e o aumento das taxas de infecções, mortalidade, quedas, PAV, extubação acidental e tempo de internação.

Conclui-se que, no cuidado a pacientes críticos, internados em UTI-A, para prevenir complicações, reduzir gastos e custos e, desenvolver cuidados de qualidade; a equipe de enfermagem deve ser dimensionada de acordo com a gravidade 
e a necessidade da clientela porque, o adequado dimensionamento da equipe de enfermagem influencia na qualidade do cuidado prestado em UTI e também, na ocorrência de eventos adversos aos pacientes críticos.

Espera-se que os resultados deste estudo forneçam subsídios para discussões e novas investigações voltadas aos processos de avaliação, planejamento e adequação do quadro de pessoal de enfermagem em UTI, com o escopo de melhorar ou manter a qualidade do cuidado prestado ao paciente crítico visto que, o cuidado humano é a essência e foco da enfermagem.

Ressalta-se que os resultados desta pesquisa apresentam limitações por advirem exclusivamente de estudos estrangeiros. Desse modo, é importante que as conclusões apresentadas, sejam transpostas e adaptadas para a realidade das UTIs brasileiras.

\section{REFERÊNCIAS}

1. Gallesio AO, Ceraso D, Palizas F. Improving quality in the intensive care unit setting. Crit Care Clin. 2006 Jul; 22(3):547-71.

2. Mezomo JC. Gestão da qualidade na saúde: princípios básicos. São Paulo (SP): Manole; 2001.

3. Padilha KG, Sousa RMC, Garcia PC, Bento ST, Finardi EM, Hatarashi RHK. Nursing workload and staff allocation in an intensive care unit: a pilot study according to Nursing Activities Score (NAS). Intensive Crit Care Nurs. 2010 Dec; 26(2):108-13.

4. Queijo AF, Padilha KG. Instrumento de medida da carga de trabalho de enfermagem em Unidade de Terapia Intensiva: Nursing Activities Score (N.A.S.). Rev Paul Enferm. 2004 Abr-Jun; 23(2):114-22.

5. Brasil, Ministério da Saúde [internet]. Brasília, DF: Ministério da Saúde; 1998 [acesso em 2010 dez 17]. Política nacional do paciente crítico. In: Consulta Pública no . 03. Disponível em: http:/ / dtr2001.saude.gov. $\mathrm{br} / \mathrm{sas} /$ PORTARIAS/Port2005/PT-03-CONS.htm

6. Brasil, Conselho Federal de Enfermagem [legislação na Internet]. Rio de Janeiro: Conselho Federal de Enfermagem; 2004 [acesso 2009 Dez 12]. Resolução COFEN n. ${ }^{\circ}$ 293/2004. Disponível em: http:/ / www. portalcofen.com.br

7. Mendes KDS, Silveira RCCP, Galvão CM. Revisão integrativa: método de pesquisa para a incorporação de evidências na saúde e na enfermagem. Texto Contexto Enferm. 2008 Out-Dez; 17(4):758-64.

8. Ducci AJ, Krokscz DVC, Bento ST, Padilha KG, Kimura M, Miyadahira AMK. Produção científica brasileira de enfermagem em terapia intensiva de 1995 a 2004 Acta Paul Enferm. 2007 Jan; 20(2):216-22

9. Carayon P, Gürses AP. A human factors engineering conceptual framework of nursing workload and patient safety in intensive care units. Intensive Crit Care Nurs. 2005 Oct; 21(5):284-301

10. Feldmann PR O atraso tecnológico da América Latina como decorrência de aspectos geográficos e de fatores microeconômicos interligados. Econ Soc. 2009 Abr; 18(1):119-39;

11. Dimick JB, Swoboda SM, Pronovost PJ, Lipsett PA. Effect of nurse-to-patient ratio in the intensive care unit on pulmonary complications and resource use after hepatectomy. Am J Crit Care. 2001 Nov; 10(6):376-82.

12. Amaravadi RK, Dimick JB, Pronovost PJ, Lipsett PA. ICU nurse-to-patient ratio is associated with complications and resource use after esophagectomy. Intensive Care Med. 2000 Dec; 26 (12):1857-62.

13. Pronovost PJ, Dang D, Dorman T, Lipsett PA, Garrett E, Jenckes M, et al. Intensive Care Unit Nurse Staffing and the Risk for Complications after Abdominal Aortic Surgery. Eff Clin Pract. 2001 SepOct; 4(5):199-206.

14. Dunton N, Gajewski B, Taunton RL, Moore J. Nurse staffing and patient falls on acute care hospital units. Nurs Outlook. 2004 Jan-Feb; 52(1):53-9

15. Hugonnet S, Chevrolet JC, Pittet D. The effect of workload on infection risk in critically ill patients. Crit Care Med. 2007 Jan; 35(1):76-81

16. Hugonnet S, Villavece A, Pittet D. Nurse staffing level and nosocomial infections: empirical evaluation of the case-crossover and case-time-control designs. Am J Epidemiol. 2007 Jun; 165(11):1321-7

17. Hugonnet S, Uçkay I, Pittet D. Staffing level: a determinant of late-onset ventilator-associated pneumonia. Crit Care. 2007 Aug; 11(4):R80

18. Pilcher J, Odele M. Position statement on nurse patient ratio in critical care. Nurs Stand. 2000 Dec; 15(12):38-41

19. Ferdinande P. Recomendations on minimal requirements for Intensive Care Departments. Intensive Care Med. 1997 Feb; 23(2):226-32

20. Thungjaroenkul P, Kunaviktikul W, Jacobs P, Cummings GG, Akkadechanunt T. Nurse staffing and cost of care in adult intensive care units in a university hospital in Thailand. Nurs Health Sci. 2008 Mar; 10(1):31-6

21. Needleman J, Buerhaus PI, Stewart M, Zelevinsky K, Mattke S. Nurse Staffing In Hospitals: Is There A Business Case For Quality? Health Aff. 2006 JanFeb; 25(1):204-11

22. Kiekkas P, Sakellaropoulos GC, Brokalaki H, Manolis E, Samios A, Skartsani C, et al. Association Between Nursing Workload and Mortality of Intensive Care Unit Patients. J Nurs Scholarsh. 2008 May; 40(4):385-90

23. Castellões TMFW, Silva LD. Ações de enfermagem para a prevenção da extubação acidental. Rev Bras Enferm. 2009 Jul-Ago; 62(4):540-5 
24. Lisboa T, Faria M, Hoher JA, Borges LAA, Gómez J, Schifelbain L, et al . Prevalência de infecção nosocomial em Unidades de Terapia Intensiva do Rio Grande do Sul. Rev Bras Ter Intensiva. 2007 Out-Dez; 19(4):414-20.
25. Bittar D B, Pereira L V, Lemos R C A. Sistematização da assistência de enfermagem ao paciente crítico: proposta de instrumento de coleta de dados. Texto Contexto Enferm. 2006 Out-Dez; 15(4):617-28. 